\title{
External quantum efficiency measurements used to study the stability of differently deposited perovskite solar cells
}

Cite as: J. Appl. Phys. 127, 235501 (2020); https://doi.org/10.1063/5.0011503

Submitted: 22 April 2020 . Accepted: 30 May 2020 . Published Online: 17 June 2020

Pedro Hierrezuelo-Cardet, Anderzon F. Palechor-Ocampo (D), Jorge Caram (D), Federico Ventosinos (D), Daniel Pérez-del-Rey (D), Henk J. Bolink (D), and Javier A. Schmidt (D)
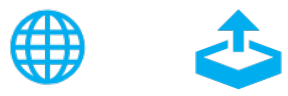

\section{Lock-in Amplifiers up to $600 \mathrm{MHz}$}
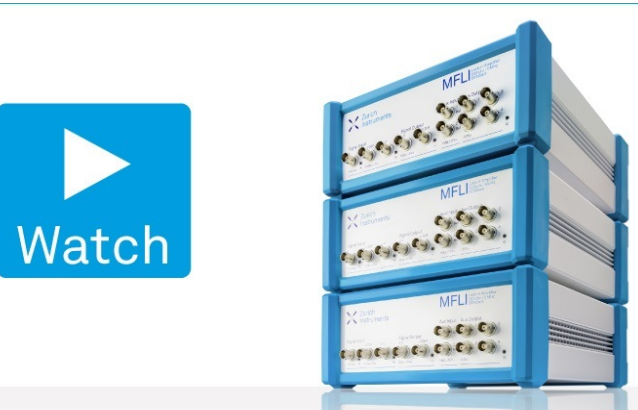


\title{
External quantum efficiency measurements used to study the stability of differently deposited perovskite solar cells
}

\author{
Cite as: J. Appl. Phys. 127, 235501 (2020); doi: 10.1063/5.001 1503 \\ Submitted: 22 April 2020 - Accepted: 30 May 2020 . \\ Published Online: 17 June 2020
}

Pedro Hierrezuelo-Cardet, ${ }^{7}$ Anderzon F. Palechor-Ocampo, ${ }^{1}$ (D) Jorge Caram, ${ }^{1,2}$ (iD Federico Ventosinos, ${ }^{1,2}$ (iD Daniel Pérez-del-Rey, ${ }^{3}$ (D) Henk J. Bolink, ${ }^{3}$ (D) and Javier A. Schmidt ${ }^{1,2, a) ~(D) ~}$

\author{
AFFILIATIONS \\ ${ }^{1}$ Instituto de Física del Litoral (IFIS-Litoral), CONICET-UNL, Güemes 3450, S3000GLN Santa Fe, Argentina \\ ${ }^{2}$ Facultad de Ingeniería Química, Universidad Nacional del Litoral, Santiago del Estero 2829, S3000AOM Santa Fe, Argentina \\ ${ }^{3}$ Instituto de Ciencia Molecular, Universidad de Valencia, C/Catedrático J. Beltrán 2, 46980 Paterna, Spain
}

Note: This paper is part of the special collection on Hybrid Organic-Inorganic Halide Perovskites

a) Author to whom correspondence should be addressed: javier.schmidt@santafe-conicet.gov.ar

\begin{abstract}
The instability exhibited by perovskite solar cells when exposed to the environment under illumination is one of the major obstacles for the entry of perovskite technology in the photovoltaic market. In this work, we use the external quantum efficiency (EQE) technique to study the photoinduced degradation of two types of solar cells having $\mathrm{CH}_{3} \mathrm{NH}_{3} \mathrm{PbI}_{3}$ as an absorber layer: one deposited by spin coating with an $n-i-p$ architecture and the other deposited by evaporation with an inverted $p$-i- $n$ structure. We also study the effect of different encapsulants to protect the cells against atmospheric agents. We find that EQE provides information regarding the areas of the cell most susceptible to degradation, in addition to providing an estimate of the optical gap and the Urbach energy of the absorbent material. We confirm that the combined action of illumination and the environment markedly accelerate the degradation, which is reflected in the deterioration of all the parameters of the cell. The rear part of the cell is the first region to suffer the light-induced degradation. On the other hand, the cells deposited by evaporation and with a good encapsulation process are highly stable since after $30 \mathrm{~h}$ of exposure just a small spectral change is noticed in the red/infrared region of the EQE spectrum.
\end{abstract}

Published under license by AIP Publishing. https://doi.org/10.1063/5.0011503

\section{INTRODUCTION}

Perovskite solar cells (PSCs) are a promising technology for low-cost and high efficiency photovoltaic devices. PSCs have gained significant interest and importance over the past few years with independently verified efficiencies now surpassing $25 \%$, ${ }^{1,2}$ as well as promising performance in light-emitting diodes, ${ }^{3}$ photodetectors, ${ }^{4}$ and lasers. ${ }^{5}$ PSCs also have significant advantages, such as being processed from different scalable processes like spraydeposition, ${ }^{6}$ spin coating, ${ }^{7}$ and vacuum deposition, ${ }^{8}$ among others. Unfortunately, many perovskite materials have relatively limited operational stability, especially compared to silicon, which is reported to have a relative mean degradation of $0.8 \%$ per year. ${ }^{9}$ It is, therefore, important to have a variety of techniques to study degradation mechanisms that limit operational stability.
Meng et al. have highlighted the need to address the stability issue of PSCs under environmental factors including light, temperature, and humidity. ${ }^{10}$ The stability under illumination at different temperatures was studied by Holzhey et al. ${ }^{11}$ Joshi et al. studied the light-induced degradation under inert atmosphere. ${ }^{12}$ Christians et al. recently demonstrated that replacing $\mathrm{TiO}_{2}$ with $\mathrm{SnO}_{2}$ as an electron transport layer enables a stable operation for at least $1000 \mathrm{~h}$ under full spectrum irradiation. ${ }^{13}$ Several studies demonstrated that the combination of light and oxygen is particularly harmful to the performance of PSCs. ${ }^{14}$ Recent reviews on the subject of degradation of PSCs can be found in Refs. 15-17.

External quantum efficiency (EQE) is a dimensionless parameter calculated as the number of electrons that exit the device divided by the number of incident photons at each wavelength. 
The photocurrent is integral over the wavelength of the product of the measured EQE with the illumination spectrum, typically AM1.5 global for terrestrial solar cells. Therefore, EQE can be used to determine the losses responsible for reducing the measured short circuit current density $\left(\mathrm{J}_{\mathrm{SC}}\right)$ from the maximum achievable photocurrent. EQE measurements have been used to study the effect of different compositions on the response of PSCs, ${ }^{18}$ different thicknesses of the electron transport layer, ${ }^{19}$ the effect of intermediate layers, ${ }^{20}$ or the segregation of phases, ${ }^{21}$ among others. However, only a few studies have used EQE measurements to follow the lightinduced degradation of PSCs. ${ }^{22}$

The focus of this work is to show that the EQE technique permits one to elucidate the effect of illumination on the performance of PSCs. We study the stability under the illumination of three different types of PSCs, one fabricated by spin coating and two deposited by vacuum evaporation. In all cases, we concentrate on the hybrid perovskite $\mathrm{CH}_{3} \mathrm{NH}_{3} \mathrm{PbI}_{3}$ (MAPI). The devices obtained by spin coating have an $n-i-p$ architecture and are not encapsulated (cells type-A). On the other hand, vacuum-deposited PSCs have a $p-i-n$ structure and differ only in the type of encapsulation adhesive used to connect an aluminum barrier sheet. In one case, an acrylic glue deposited over the full area of the stack is used (sample type-B), whereas in the other case a UV-curable epoxy resin is applied only on the bare glass outside of the active device area (sample type-C).

Solar cell preparation is described in Sec. II A. Degradation under light was carried out as described in Sec. II B. In Secs. II C and II D, we present the experimental methods, current densityvoltage $(J-V)$ scans, and EQE scans, used to explore degradation mechanisms on the three types of samples. In Sec. III, we discuss the main results, and finally, we present our conclusions in Sec. IV.

\section{EXPERIMENTAL METHODS}

In this work, to highlight the variability in performance and behavior of different device architectures, two different solar cell structures are studied: a regular $n-i-p$ device not encapsulated (sample type-A) and two inverted $p-i-n$ devices with two different encapsulation methods (samples type-B and type-C).

\section{A. Solar cell preparation}

Type-A devices have a final structure FTO $(380 \mathrm{~nm}) /$ compact-SnO $2(20 \mathrm{~nm}) / \mathrm{MAPI}(320 \mathrm{~nm}) /$ Spiro-OmeTAD $(190 \mathrm{~nm}) / \mathrm{Au}$ $(80 \mathrm{~nm})$, where FTO is fluorine-doped tin oxide and Spiro-OmeTAD is $2,2^{\prime}, 7,7^{\prime}$-tetrakis[ $N, N$-di(4-methoxyphenyl)amino]-9,9' -spirobifluorene.

The devices were prepared at the Institute of Advanced Materials, University Jaume I, Castelló, Spain. FTO-covered glasses, without any meso-porous layer, were used as substrates. All the processes were carried out inside a glovebox with oxygen and water levels below $3 \mathrm{ppm}$. The substrates were partially etched with zinc powder and $\mathrm{HCl}$ (2M) to remove a fringe of FTO. Then, the substrates were cleaned with Hellmanex solution and rinsed with Milli-Q water and ethanol, followed by $15 \mathrm{~min}$ of sonication in three steps with acetone, ethanol, and isopropanol. The $\mathrm{SnO}_{2}$ compact (blocking) layer was deposited onto the substrate by spin coating from a colloidal solution. The perovskite precursor solution was spin-coated using toluene as antisolvent, followed by heating at $100^{\circ} \mathrm{C}$ for $3 \mathrm{~min}$. For the hole transport layer (HTL), a solution of Spiro-OMeTAD was prepared by dissolving $72.3 \mathrm{mg}$ of $2,2^{\prime}, 7,7^{\prime}$-tetrakis $(N$, $N^{\prime}$-di-p-methoxyphenylamine)-9, $9^{\prime}$-spirobifluorene in $1 \mathrm{ml}$ of chlorobenzene, $28.8 \mu \mathrm{l}$ of 4-tert-butylpyridine, and $17.5 \mu \mathrm{l}$ of a stock solution of $520 \mathrm{mg} / \mathrm{ml}$ of lithium bis-(trifluoromethylsulfonyl) imide in acetonitrile, as additives. The perovskite film was then coated with the HTL solution by dynamically spin coating at $4000 \mathrm{rpm}$ for $30 \mathrm{~s}$. Finally, $100 \mathrm{~nm}$ of gold was thermally evaporated on top of the device to form the metal electrode contacts. For more details on the preparation of the type-A devices, see the work of Aranda et al.

Inverted $p-i-n$ devices, type- $\mathrm{B}$ and $-\mathrm{C}$ samples, were prepared at the Institute of Molecular Science, Valencia University, Paterna, Spain, as described in Ref. 8. The final structure of the devices is $\mathrm{ITO} / \mathrm{MoO}_{3}$ $(5 \mathrm{~nm}) / \mathrm{TaTm}(10 \mathrm{~nm}) / \mathrm{MAPI}(590 \mathrm{~nm}) / \mathrm{C}_{60}(25 \mathrm{~nm}) / \mathrm{BCP}(8 \mathrm{~nm}) / \mathrm{Ag}$, where ITO is indium tin oxide, TaTm is $\mathrm{N} 4, \mathrm{~N} 4, \mathrm{~N} 4^{\prime}, \mathrm{N}^{\prime}$-tetra

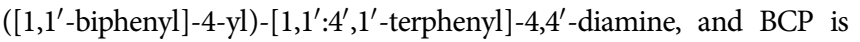
bathocuproine. In brief, ITO prepatterned substrates were cleaned following a standard procedure and transferred to a vacuum chamber evacuated to a pressure of $10^{-6}$ mbar. The vacuum chamber was equipped with six temperature-controlled evaporation sources (Creaphys) fitted with ceramic crucibles. The substrate holder to evaporation source distance was approximately $20 \mathrm{~cm}$. The materials were sublimed at temperatures ranging from 60 to $>300^{\circ} \mathrm{C}$, and the evaporation rate was controlled by separate sensors. In general, the deposition rate for TaTm and $\mathrm{C}_{60}$ was $0.5 \AA \mathrm{s}^{-1}$ and $0.2-0.3 \AA \mathrm{s}^{-1}$ for the thinner BCP. For the perovskite deposition, MAI and $\mathrm{PbI}_{2}$ were coevaporated by measuring the deposition rate of each material in a different sensor, and obtaining the total perovskite thickness in a third one, leading to a $590 \mathrm{~nm}$-thick perovskite. $\mathrm{MoO}_{3}$ and $\mathrm{Ag}$ were evaporated in a second vacuum chamber. All vacuum chambers are inside inert atmosphere nitrogen gas filled gloveboxes with water and oxygen levels below $0.1 \mathrm{ppm}$. The area of the devices is around $5 \mathrm{~mm}^{2}$. Type-B devices were encapsulated by depositing an acrylic glue over the full area of the stack and placing a thin aluminum cap on top. For type-C devices, we used "Encapsulation Epoxy for Photovoltaics and OLEDs" from Ossila (code E132) to adhere a thin aluminum cap to the free glass surface on the edge of the ITO coated glass substrates. The cells were illuminated with UV light for $20 \mathrm{~min}$ to ensure the curing of the adhesive. The encapsulation processes were carried out in an inert atmosphere glovebox.

\section{B. Degradation under illumination}

The devices were illuminated with a tungsten-halogen lamp. The irradiance was set to $100 \mathrm{~mW} \mathrm{~cm}^{-2}$ using a calibrated silicon photodiode. To exclude additional degradation induced by heating,

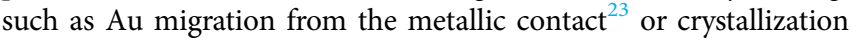
of the hole transport layer (spiro-MeOTAD in type-A devices), the temperature of the devices was kept at $40 \pm 4{ }^{\circ} \mathrm{C}$. This was achieved by cooling the devices with a fan and illuminating through a shadow mask with the area of the cell. The experimental procedure involved the following steps. First, the EQE and the dark $I-V$ curves were measured at room temperature. Then, the cell was illuminated and the light $I-V$ curve was measured. The beginning of the illumination is $\mathrm{t}=0$ in this experiment; as the measurement of the $I-V$ curve takes approximately $1 \mathrm{~min}$, the first $I-V$ curve is considered as $\mathrm{t}=1 \mathrm{~min}$. Without interrupting the illumination, the 
second $I-V$ curve was measured after $3 \mathrm{~min}$. Next, the illumination was interrupted and the cell was kept $2 \mathrm{~min}$ in the dark under forced-air convection cooling to recover room temperature. The temperature evolution was measured with a platinum resistor attached to the surface of the cell. The EQE curves were always measured at room temperature. After the EQE measurement, illumination was resumed and the illumination time was restarted. We have found that it takes approximately $3 \mathrm{~min}$ of illumination for the temperature to stabilize at $\sim 40^{\circ} \mathrm{C}$.

\section{C. $I-V$ characterization}

The $I-V$ curves were measured with a Siglent SDM 3055 multimeter and a GW-Instek GPD-3303S voltage source in the dark and under illumination with the same tungsten-halogen lamp used for light-induced degradation. In all $I-V$ measurements, a voltage sweep from 1.2 to $-0.1 \mathrm{~V}$ with a scan rate of $60 \mathrm{mV} \mathrm{s}^{-1}$ was used.

\section{External quantum efficiency}

The basic idea in an external quantum efficiency experiment is to know the ratio between the number of photogenerated carriers per incident photons, as a function of the wavelength $\lambda$, under short circuit conditions,

$$
\operatorname{EQE}(\lambda)=\frac{n_{e}(\lambda)}{n_{p h}(\lambda)}
$$

One practical way to deduce this quantity is to measure the short circuit current of a calibrated photodiode with known EQE, and then measuring the short circuit current of the unknown device, obtaining its $\mathrm{EQE}$ as

$$
E Q E_{u n k}(\lambda)=\frac{I_{u n k}(\lambda)}{I_{d}(\lambda)} E Q E_{d}(\lambda) .
$$

In this equation, $I(\lambda)$ is the short circuit current measured at each wavelength, the subscript unk refers to the unknown device, while $d$ is for the calibrated photodiode. It is important to note that, since we are dividing by the current of the calibrated diode if the light source used in the experiment does not change between measurements, then EQE is independent of the lamp used. This is important because the short circuit current density under standard test conditions can be derived from EQE as

$$
J_{s c}=q \int_{0}^{\infty} \Phi(\lambda) E Q E(\lambda) d \lambda .
$$

In this equation, $q$ is the electron charge and $\Phi$ is the photon flux corresponding to the AM1.5 spectrum. ${ }^{24}$

The experimental setup that we use is shown in Fig. 1. As it can be seen, we use a Xenon arc lamp as a light source, which goes through a computer-controlled double pass monochromator. Since the measurement of a dc current can be problematic due to noise, we use lock-in amplifiers together with current/voltage amplifiers to have a better signal to noise ratio. The addition of a second reference diode is done in order to correct the measurement by small changes in the intensity of the illumination source (that may happen due to temperature variations, for example). A comprehensive revision of the EQE technique for different types of solar cells can be found in Ref. 25. In order to measure EQE curves, we used $10 \mathrm{~nm}$ steps from 400 to $850 \mathrm{~nm}$. Each point was measured ten times, and a waiting time of $5 \mathrm{~s}$ was set between different wavelengths. Each data point shown in the following figures is the average of ten measurements, while the error bars correspond to the statistical error. In all measurements, the temperature was $25 \pm 2{ }^{\circ} \mathrm{C}$ and no bias light was used.

\section{RESULTS AND DISCUSSION}

Type-A devices, fabricated by spin coating and unencapsulated, exhibit a poor stability as depicted in Fig. 2. Figure 2(a) shows the current density-voltage curves as a function of illumination time. In Fig. 2(b), it can be seen that the open circuit voltage $\left(\mathrm{V}_{\mathrm{OC}}\right)$ loses more than $20 \%$ after $180 \mathrm{~min}$ of illumination. On the other hand, the fill factor (FF) remains approximately constant after an initial drop. Figure 2(c) shows that the short circuit current density $\left(\mathrm{J}_{\mathrm{SC}}\right)$ decreases by a factor of $\sim 5$. As a result, the power conversion efficiency decreases to $\sim 15 \%$ of the initial value [Fig. 2(d)]. Degradation occurs in a short time period because the samples are not encapsulated, and the simultaneous action of light and oxygen from the environment degrades MAPI with the formation of $\mathrm{PbI}_{2}$ and other lead salts. ${ }^{14,26}$

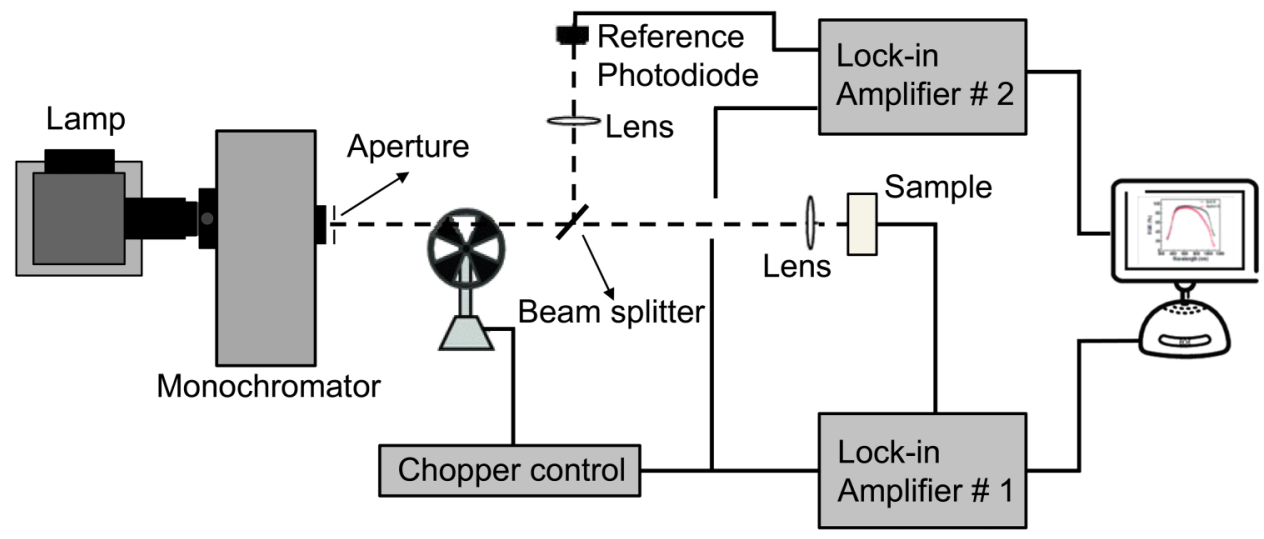

FIG. 1. External quantum efficiency experimental setup. 

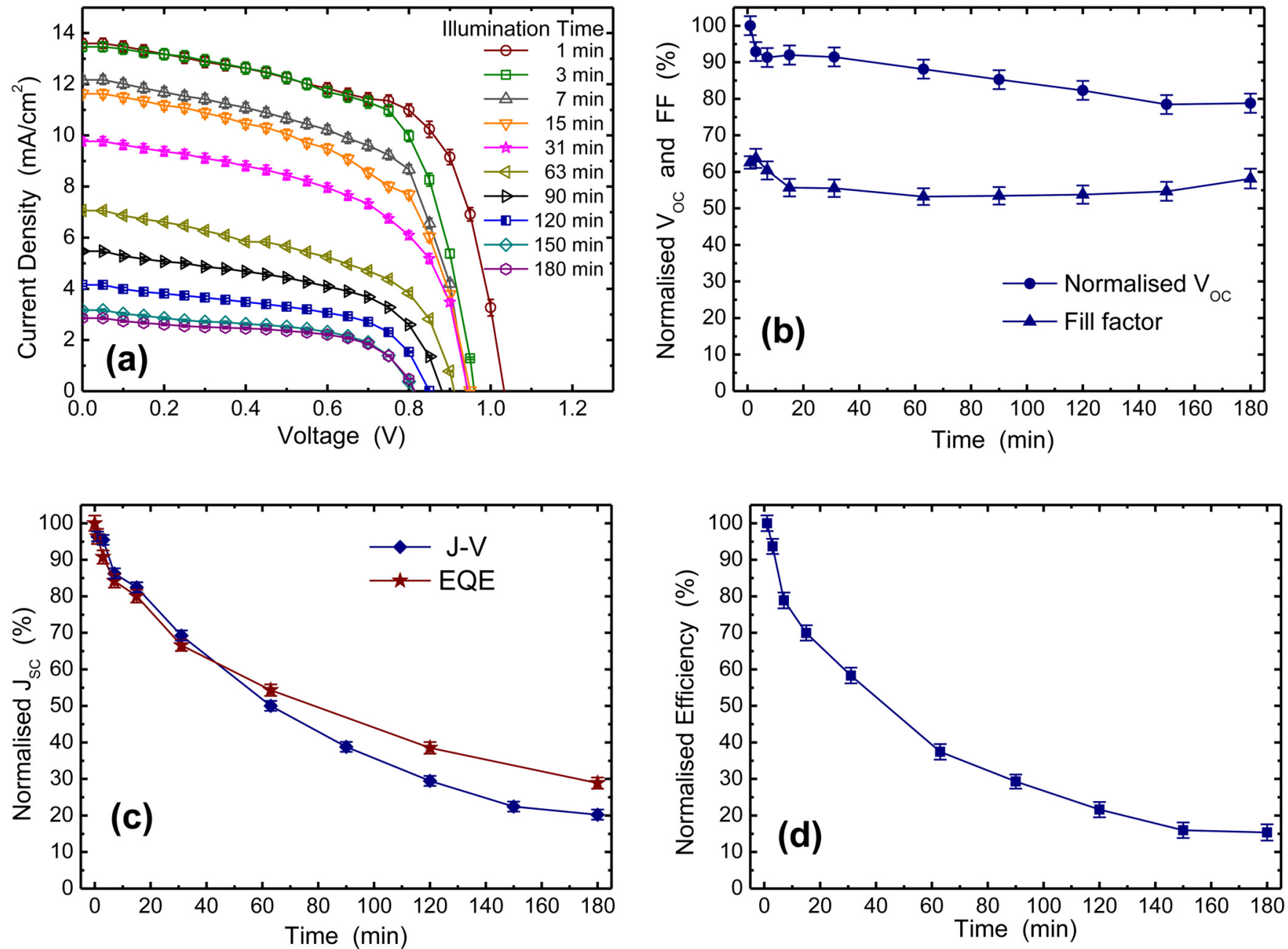

FIG. 2. Degradation as a function of time on unencapsulated PSCs obtained by the spin coating method. (a) Current density-voltage curves (J-V). (b) Normalized open circuit voltage and fill factor as a function of illumination time. (c) Normalized current densities obtained from the $J-V$ and the EQE curves as a function of time. (d) Normalized efficiency as a function of time.

In accordance with the decrease in $\mathrm{J}_{\mathrm{SC}}$, we observe a decrease in the EQE of the type-A samples, as shown in Fig. 3(a), with the maximum EQE value dropping from $64 \%$ to $20 \%$ in 180 min of illumination. The shape of the EQE curve also changes with greater impact in wavelengths above $500 \mathrm{~nm}$, as will become apparent in the analysis of Fig. 4. This behavior may be associated with an increase in the relative levels of $\mathrm{PbI}_{2}$ within the device. ${ }^{22,27}$ The use of Eq. (3) to calculate $\mathrm{J}_{\mathrm{SC}}$ from the EQE spectrums provides a good agreement with the photocurrent density obtained from $J-V$ measurements, as shown in Fig. 2(c). The difference that can be seen especially for long illumination times may be due to the fact that $J-V$ and EQE measurements are performed at slightly different temperatures, and EQE is measured without a bias light.

The EQE curves can also be used to estimate the bandgap energy $\left(E_{G}\right)$ of the absorber material of a solar cell. ${ }^{28}$ The first derivative of the EQE curves, $d(E Q E) / d \lambda$, exhibits a sharp peak in the long-wavelength region. Performing the derivative of the spectra of Fig. 3(a), a peak at $774 \mathrm{~nm}$ is observed, corresponding to $E_{G}=1.602 \mathrm{eV}$. This value agrees with the one obtained from the optical transmittance spectrum of a single MAPI layer. The open circuit voltage deficit, $V_{O C, \text { def }}$, is defined by $E_{G} / q-V_{O C}$ and is related to the carrier recombination and the absorber quality. ${ }^{28}$ From the initial $J-V$ curve of Fig. $2(\mathrm{a}), V_{O C}=1.034 \mathrm{~V}$ and $V_{O C, \text { def }}=0.568 \mathrm{~V}$ is found for these type-A cells. Moreover, an indication of the quality of the absorber material may be given by the width of the Urbach tail, $E_{U}$. The Urbach tails are comprised of electronic states distributed within the bandgap with a density exponentially increasing toward the band edges. The $E_{U}$ value for the absorber layer of a solar cell can be calculated from the long-wavelength edge of its EQE (near the band edge) using the 

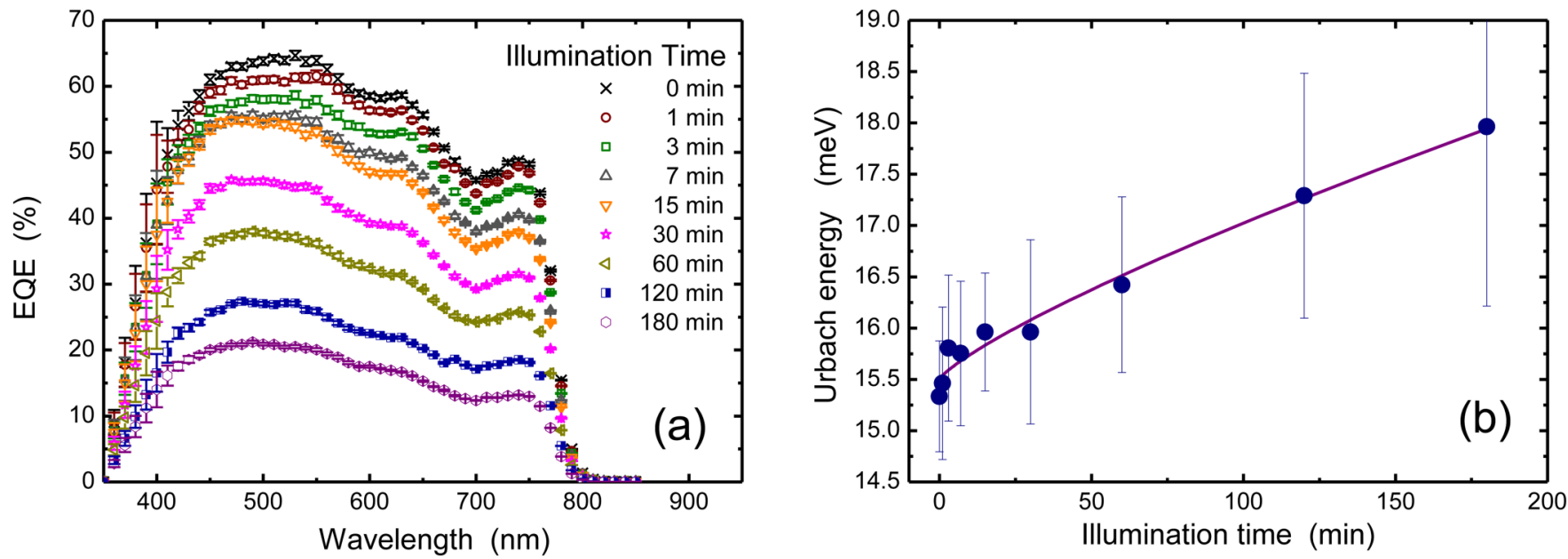

FIG. 3. Degradation as a function of illumination time studied from external quantum efficiency measurements. (a) EQE spectra for the illumination times indicated in the legend. (b) Evolution of the Urbach energy with the illumination time; the line is a guide to the eye.

expression $^{29}$

$$
\ln (E Q E)=\frac{h v}{E_{U}}+\mathbb{C}
$$

where $h v$ is the photon energy and $\mathbb{C}$ is a constant. A fit with Eq. (4) of the EQE curves of Fig. 3(a) gives the $E_{U}$ values plotted in Fig. 3(b). As can be seen, the Urbach energy tends to increase with the illumination time, in agreement with the observed deterioration of all the parameters of the solar cell.

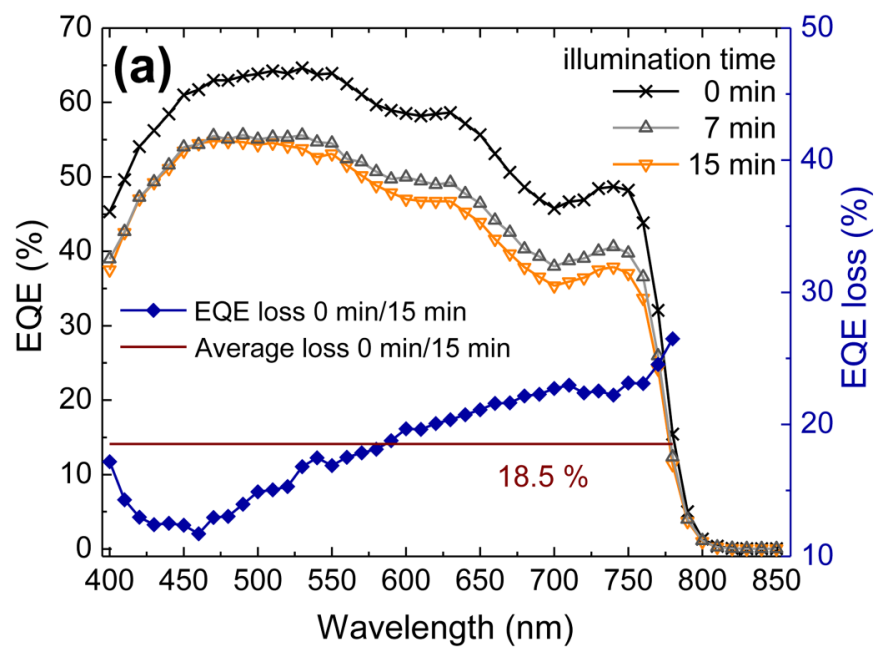

In order to distinguish between humidity and illumination, we used a reference cell from the same batch of type-A devices and having almost identical initial performance. This reference cell was exposed to the environment at the same time that device A was being illuminated, the only difference being that the reference device was kept under dark conditions, thus evidencing only the ambient-induced degradation. Figure 4 shows the comparison between a cell exposed to light and the environment [Fig. 4(a)] and the reference cell [Fig. 4(b)]. As can be seen, 15 min of combined exposure to light and air causes a similar degradation than $30 \mathrm{~h}$ of air exposure alone. Taking advantage of the possibility offered by

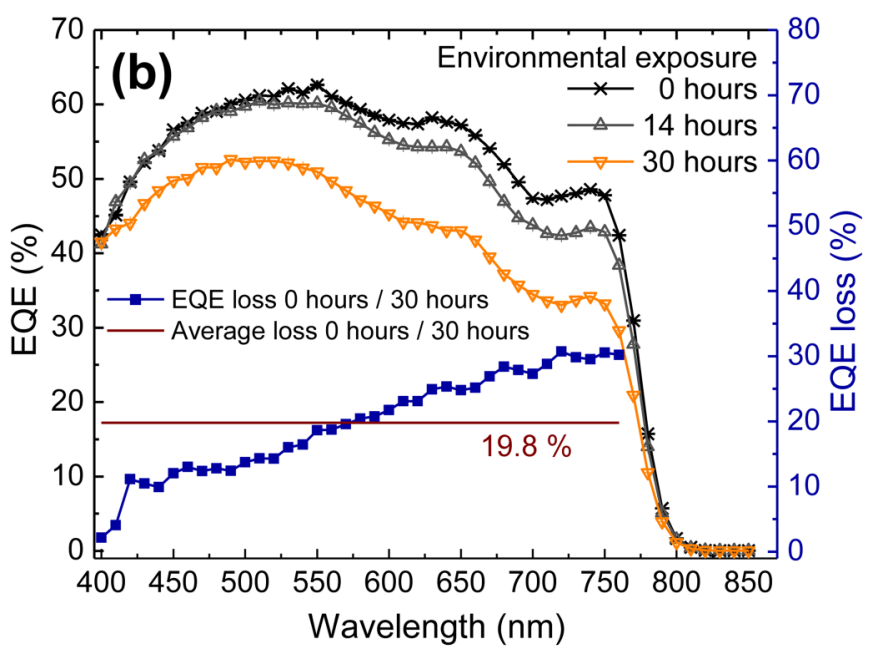

FIG. 4. External quantum efficiency (left scales) and percentage decrease relative to $t=0$ (right scales) for different time exposures: (a) cell simultaneously exposed to the environment and illuminated and (b) cell exposed only to the environment. 
EQE of obtaining spectral information, we can gain insight into the behavior of the different regions of the cell. We can see that in both cases the percentage decrease in EQE, relative to the $t=0$ spectra, have the same profile (right scales). The decay is more pronounced in the red/infrared region of the spectra close to the absorption edge. As infrared light is uniformly absorbed in the device, this region of the EQE curve provides information about what is taking place in the bulk and rear surface of the device. Considering the structure of type-A devices, this region corresponds to the MAPI layer or the MAPI/Spiro-OmeTAD interface. Since the back of these devices is exposed to the ambient, it is reasonable to assume that the degradation should start from these layers. Although the back of the cell is partially covered by gold contacts, oxygen/moisture is likely to diffuse inward from the edges of the contacts. Also, degradation may well happen first at the grain boundaries since it is in this area where the ingress is easiest. Degradation at interfaces and fairly uniformly within the bulk has been previously inferred from wavelength-dependent laser-beam mapping experiments. ${ }^{22}$ From the comparison of Figs. 4(a) and 4(b), it is clear that the illumination has a pronounced effect accelerating the ambient-induced degradation, as already pointed out by other authors. ${ }^{22}$

The EQE curves of Fig. 5(a) correspond to a type-B device, exposed to illumination under ambient conditions. The EQE technique allows us to observe the degradation of the different zones of this device, which is fabricated by vacuum evaporation and encapsulated with an acrylic glue. Comparing with Fig. 3(a), it is evident that type-B devices exhibit a considerable improvement in stability over type-A devices. The degradation takes place on a time scale of hours for type-B devices, compared to minutes for type-A. This is due to (i) type-B solar cells are encapsulated, avoiding large incorporation of humidity and oxygen in the active layer; (ii) the vacuum evaporation method favors homogeneity, reduces the density of defects, and eliminates the presence of water and organic solvents; and (iii) the use of passivating layers reduces the interface defects. These differences are essential in obtaining good PSCs. ${ }^{30}$ In type-A devices, the MAPI layer was fabricated by spin coating, a method that does not guarantee homogeneity in the deposition. Also, evaporation is known to produce layers with a lower defect concentration. ${ }^{31,32}$ Finally, the encapsulant is a fundamental part of any solar device of this type, the absence of which expose the solar cells to degradation phenomena.

The EQE curves of Fig. 5(a) were also used to estimate $E_{G}$ for the absorber material of this type- $\mathrm{B}$ device. ${ }^{28}$ The first derivative $d(E Q E) / d \lambda$ exhibits a sharp peak for $770 \mathrm{~nm}$, corresponding to $E_{G}=1.61 \mathrm{eV}$. The open circuit voltage for this device is $V_{O C}=1.059 \mathrm{~V}$, meaning an open circuit voltage deficit $V_{O C, \text { def }}=0.551 \mathrm{~V}$ for these type- $B$ cell slightly lower than the value obtained for type-A devices. The $E_{U}$ value for the absorber layer of this solar cell has also been calculated from the long-wavelength edge of its EQE using Eq. (4). ${ }^{29}$ The $E_{U}$ values are plotted in Fig. 5(b) as circles. It can be seen that there is a sharp increase in $E_{U}$ for the first $45 \mathrm{~min}$ of illumination, followed by a slight increase as the degradation evolves. Moreover, the $E_{U}$ values are lower than those obtained for type-A devices [Fig. 3(b)], indicating a higher quality for the absorber material.

As can be seen in Fig. 5(a), the first $45 \mathrm{~min}$ of exposure produce a detriment in the EQE for wavelengths lower than $500 \mathrm{~nm}$ and an improvement for the rest of the spectrum. This initial effect disappears after hours of exposure when the degradation extends to the whole spectrum. To gain insight into the behavior of the different regions of the cell, we present in Fig. 6, the behavior of this cell after $45 \mathrm{~min}$ [Fig. 6(a)] and $10.5 \mathrm{~h}$ [Fig. 6(b)] of illumination. In both cases, the right scales correspond to the percentage decrease in $\mathrm{EQE}$, relative to the $t=0$ spectra. The EQE spectrum after the first $45 \mathrm{~min}$ of illumination [Fig. 6(a)] presents a deterioration in the front region of the device of around $8.6 \%$, but
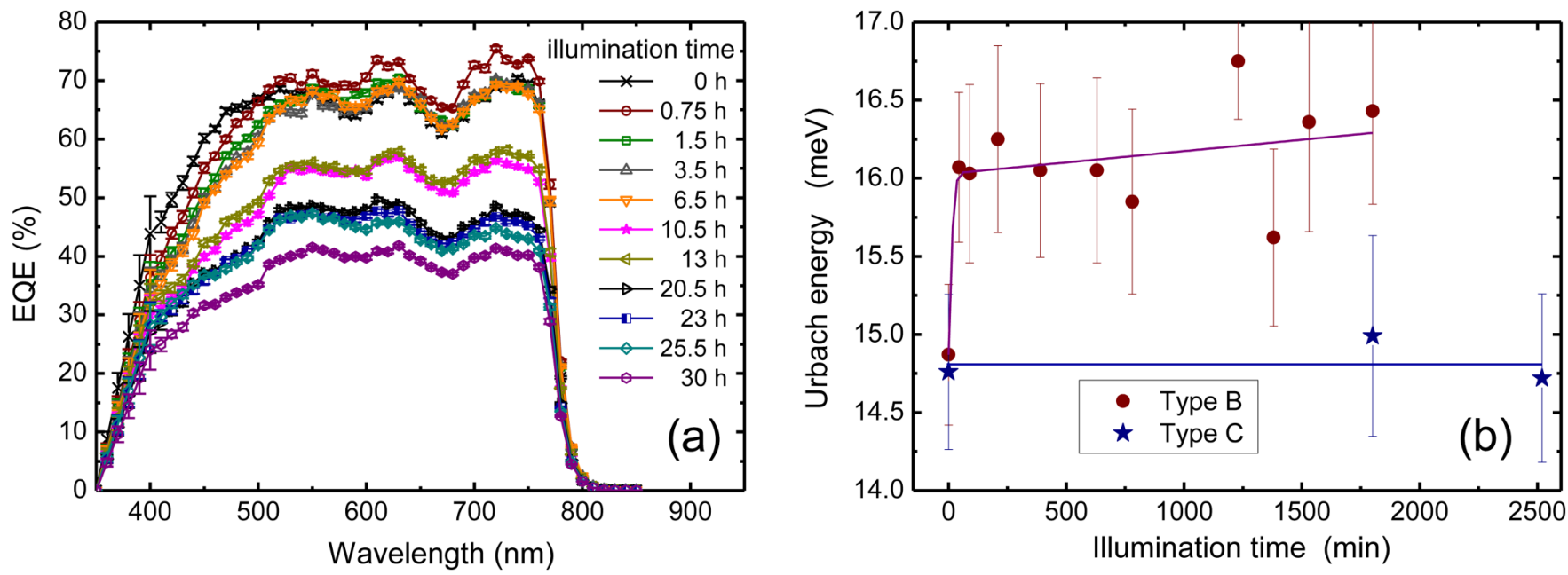

FIG. 5. Light-induced degradation of PSCs obtained by vacuum evaporation: (a) EQE spectra of type-B devices for the illumination times indicated in the legend and (b) evolution of the Urbach energy with the illumination time, for type-B (circles) and type-C (stars) devices; the lines are a guide to the eye. 

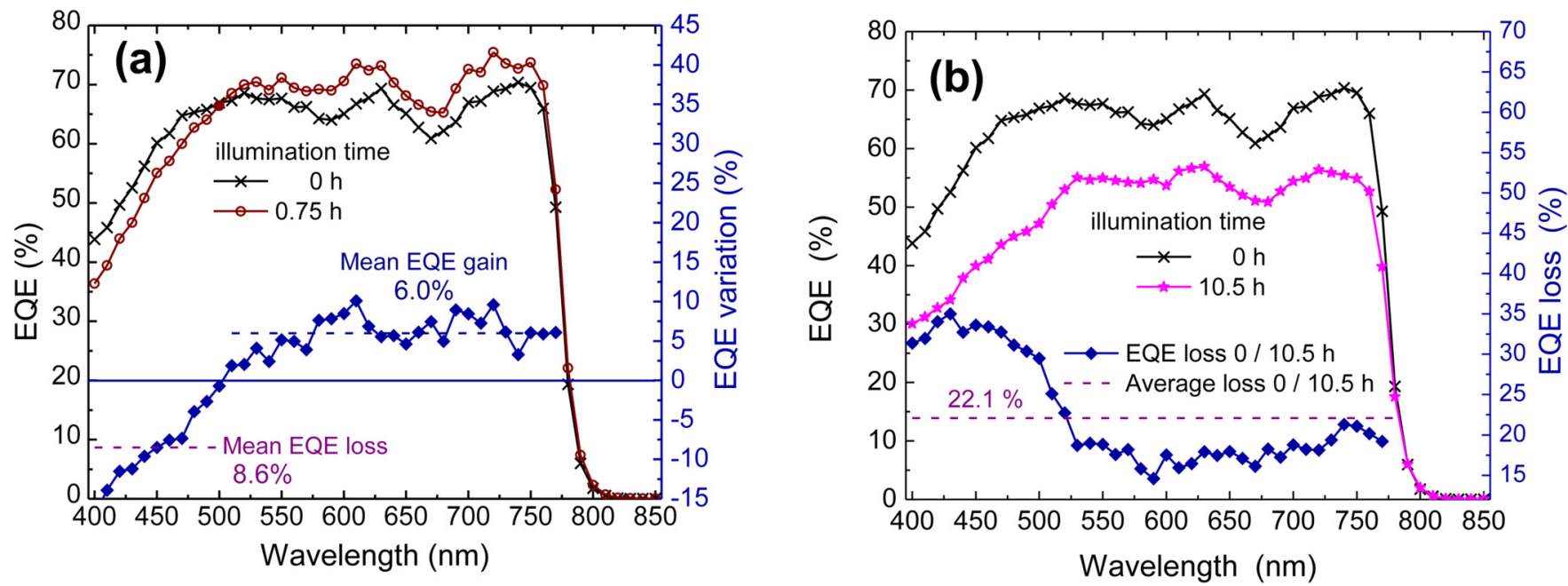

FIG. 6. External quantum efficiency (left scales) and percentage decrease relative to $t=0$ (right scales) for type-B cells (a) after 45 min of illumination and (b) after $10.5 \mathrm{~h}$ of illumination, in both cases under ambient conditions.

at the same time a gain of $\sim 6 \%$ in the rest of the device. Although the solar spectrum is absorbed throughout the whole cell, the more energetic (or short wavelength, UV) photons are absorbed mostly at the front of the device. These energetic photons may cause irreversible damage to the $\mathrm{Pb}-\mathrm{I}_{2}$ bonds on the perovskite layer. ${ }^{33}$ The improvement in the rest of the device may be associated with ion migration, specially $\mathrm{I}^{-}$species, as suggested by some authors. ${ }^{34}$ This behavior would be favored by the initial increase in temperature when the samples are exposed to illumination for the first time. As the exposure time increases [Fig. 6(b)], a deterioration of the EQE is observed in the whole range of wavelengths, although the loss in the front region is still more important. We recall that in these $p-i-n$ structures the front region of the device corresponds to the $\mathrm{MoO}_{3} / \mathrm{TaTm} / \mathrm{MAPI}$ interfaces.

One of the ways to reduce the degradation effects discussed here, such as the incorporation of oxygen and water molecules, is with better encapsulation. Figure 7 shows the behavior of type- $C$ devices.
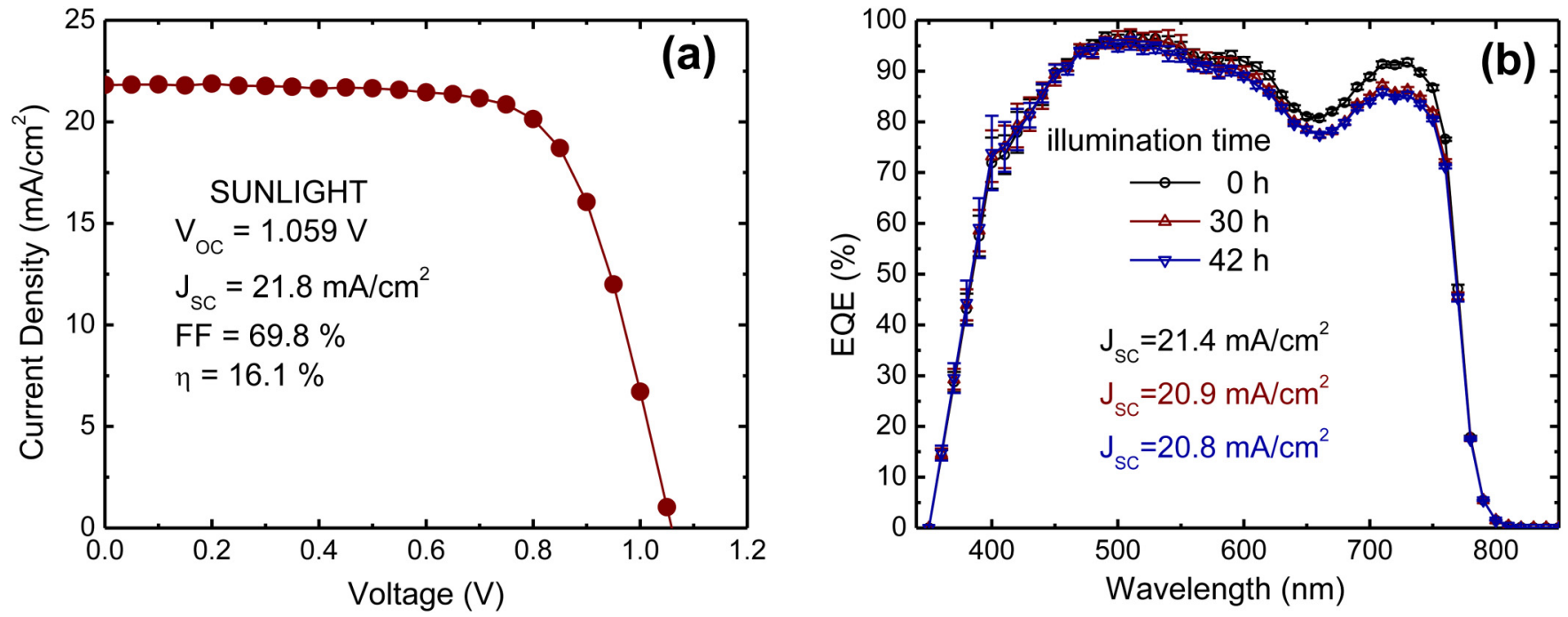

FIG. 7. Degradation as a function of time for type-C PSCs obtained by the vacuum evaporation method. (a) Current density-voltage curve (J-V), the characteristic parameters of the cell are indicated in the legend. (b) EQE for different illumination times, the short circuit current densities obtained from Eq. (3) are indicated in the legend. 
Type- $C$ devices were exposed to direct sunlight for the measurement of the $I-V$ curve. The irradiance of $100 \mathrm{~mW} / \mathrm{cm}^{2}$ was measured with a Kipp \& Zonen CMP6 pyranometer. Figure 7(a) allows us to obtain the electrical parameters from which we can determine the fill factor and the efficiency of the device. Observing the values obtained for this type of cell, we are in the presence of a good cell with an efficiency of $16.1 \%$. Using again the EQE technique, Fig. 7 (b) shows that in this device there is a general improvement in charge collection. The external quantum efficiency is around $72 \%$ for $400 \mathrm{~nm}$ and reaches almost $100 \%$ for $500 \mathrm{~nm}$. This is an indication of better surface passivation and a much lower recombination rate compared to the other devices. The EQE technique shows a much more stable device to the exposure of light and the environment; after $30 \mathrm{~h}$ of exposure, the first spectral changes are hardly observed in the red/infrared region of the spectra. This degradation is only appreciable by the EQE technique, since the $J-V$ curves are almost identical, being the decrease of the current density of $2.8 \%$. The $\mathrm{J}_{\mathrm{SC}}$ values obtained from Eq. (3) agree well with the value measured from the $J-V$ curve, cross validating the results. The $E_{U}$ values of type- $C$ device, obtained fitting with Eq. (4), the EQE curves of Fig. 7(b), are plotted in Fig. 5(b) as stars. It can be seen that $E_{U}$ remains low even when the sample is illuminated under ambient conditions, meaning that the encapsulant used for this device effectively reduces the degradation. The values obtained for $E_{U}$, around $14.8 \mathrm{meV}$, are within the range of values calculated from data available in literature studies. ${ }^{28,35,36}$

There are several reasons for the enhanced stability of the evaporated and encapsulated devices compared to type-A devices. The absence of residual solvents will reduce the ionic mobility in the perovskite film, and the smooth perovskite film allows for the use of thin charge extraction layers that, therefore, do not need to be oxidized. But probably the main reason is the absence of water, which is known to form an equilibrium (acid-base) reaction with the methylammonium cations. Upon proton transfer from the MA to the water molecules, the resulting methylamine escapes as a gas which leads to the disintegration of the perovskite and the formation of $\mathrm{PbI}_{2}$. As the bandgap of $\mathrm{PbI}_{2}$ is larger than that of the perovskite, the formation of it near a charge extracting interface would lead to an energy barrier for charge extraction that would be observed as a decrease in the fill factor of the solar cells. Therefore, we believe that the higher purity of the evaporated materials and the absence of water and organic solvents for thin-film formation contribute to the improved stability of these devices.

\section{CONCLUSIONS}

The EQE technique has been used to observe the spectral changes that occur in the different regions of perovskite photovoltaic devices when exposed to the environment and to illumination with an irradiance of $100 \mathrm{~mW} / \mathrm{cm}^{2}$. Type-A devices, which are not encapsulated, show a rapid drop in their efficiency when exposed to light under ambient conditions. On the other hand, when exposed to the environment only, the decay of the EQE occurs much more slowly, losing $20 \%$ of its initial value in $30 \mathrm{~h}$. The fact that the same type of sample loses an equal amount of EQE in $15 \mathrm{~min}$ when illuminated and exposed to the environment, confirmed that light accelerates the degradation of the device. Type- $\mathrm{B}$ devices show a substantial improvement in stability compared to type-A, exhibiting even an increase in the part of the EQE spectrum in the first minutes of illumination. These devices are prepared by vacuum evaporation with an inverted $p-i-n$ architecture and are encapsulated. In the case of type- $C$ devices, with an improvement in the encapsulant, the stability is remarkable and after $30 \mathrm{~h}$ of exposure, just a small spectral change is noticed in the $\mathrm{red} /$ infrared region of the spectrum. This confirms that encapsulation is extremely important in PSCs, reducing the effects of degradation due to the incorporation of oxygen and water molecules present in the environment.

\section{ACKNOWLEDGMENTS}

The research leading to these results has received funding from the Argentinean National Scientific and Technical Research Council (CONICET) Project No. PUE 22920160100054CO, the Argentinian National Agency for Science and Technology Promotion (ANPCyT) (No. PICT-2016-1389), the Litoral National University (UNL) (No. CAI + D 50420150100108L), the Spanish Ministry of Science, Innovation and Universities (Nos. MAT2017-88821-R and EQC2018-004888-P), and the Comunitat Valenciana (No. IDIFEDER/2018/061). J.C. acknowledges support from the Institute of Advanced Materials, University Jaume I, Castelló, Spain, during his research stay.

\section{DATA AVAILABILITY}

The data that support the findings of this study are available within the article or are available from the corresponding author upon reasonable request.

\section{REFERENCES}

${ }^{1}$ See https://www.nrel.gov/pv/assets/pdfs/best-research-cell-efficiencies.20200406. pdf for information about the evolution of the best research-cell efficiencies.

${ }^{2}$ E. H. Jung, N. J. Jeon, E. Y. Park, C. S. Moon, T. J. Shin, T.-Y. Yang, J. H. Noh, and J. Seo, Nature 567, 511 (2019).

${ }^{3}$ W. Xu, Q. Hu, S. Bai, C. Bao, Y. Miao, Z. Yuan, T. Borzda, A. J. Barker, E. Tyukalova, Z. Hu, M. Kawecki, H. Wang, Z. Yan, X. Liu, X. Shi, K. Uvdal, M. Fahlman, W. Zhang, M. Duchamp, J.-M. Liu, A. Petrozza, J. Wang, L.-M. Liu, W. Huang, and F. Gao, Nat. Photonics 13, 418 (2019).

${ }^{4} \mathrm{~V}$. Venugopalan, R. Sorrentino, P. Topolovsek, D. Nava, S. Neutzner, G. Ferrari, A. Petrozza, and M. Caironi, Chem 5, 868 (2019).

${ }^{5}$ H. Zhu, Y. Fu, F. Meng, X. Wu, Z. Gong, Q. Ding, M. V. Gustafsson, M. T. Trinh, S. Jin, and X.-Y. Zhu, Nat. Mater. 14, 636 (2015).

${ }^{6}$ D. K. Mohamad, J. Griffin, C. Bracher, A. T. Barrows, and D. G. Lidzey, Adv. Energy Mater. 6, 1600994 (2016).

${ }^{7}$ C. Aranda, J. Bisquert, and A. Guerrero, J. Chem. Phys. 151, 124201 (2019).

${ }^{8}$ D. Pérez-del-Rey, L. Gil-Escrig, K. P. S. Zanoni, C. Dreessen, M. Sessolo, P. P. Boix, and H. J. Bolink, Chem. Mater. 31, 6945 (2019).

${ }^{9}$ D. C. Jordan and S. R. Kurtz, Prog. Photovolt. 21, 12 (2013).

${ }^{10}$ L. Meng, J. You, and Y. Yang, Nat. Commun. 9, 5265 (2018).

${ }^{11}$ P. Holzhey, P. Yadav, S.-H. Turren-Cruz, A. Ummadisingu, M. Grätzel, A. Hagfeldt, and M. Saliba, Mater. Today 29, 10 (2019).

${ }^{12}$ P. H. Joshi, L. Zhang, I. M. Hossain, H. A. Abbas, R. Kottokkaran, S. P. Nehra, M. Dhaka, M. Noack, and V. L. Dalal, AIP Adv. 6, 115114 (2016).

${ }^{13}$ J. Christians, P. Schulz, J. Tinkham, T. Schloemer, S. Harvey, B. Tremolet de Villers, A. Sellinger, J. Berry, and J. Luther, Nat. Energy 3, 68 (2018).

${ }^{14}$ D. Bryant, N. Aristidou, S. Pont, I. Sanchez-Molina, T. Chotchunangatchaval, S. Wheeler, J. R. Durrant, and S. A. Haque, Energy Environ. Sci. 9, 1655 (2016). 
${ }^{15}$ C. C. Boyd, R. Cheacharoen, T. Leijtens, and M. D. McGehee, Chem. Rev. 119, 3418 (2019).

${ }^{16}$ S. P. Dunfield, L. Bliss, F. Zhang, J. M. Luther, K. Zhu, M. F. A. M. van Hest, M. O. Reese, and J. J. Berry, Adv. Energy Mater. (in press).

${ }^{17}$ S. He, L. Qiu, L. K. Ono, and Y. Qi, Mater. Sci. Eng. R Rep. 140, 100545 (2020).

${ }^{18}$ C. Yi, J. Luo, S. Meloni, A. Boziki, N. Ashari-Astani, C. Grätzel, S. M. Zakeeruddin, U. Röthlisberger, and M. Grätzel, Energy Environ. Sci. 9, 656 (2016).

19 J. H. Heo, S. H. Im, J. H. Noh, T. N. Mandal, C.-S. Lim, J. A. Chang, Y. H. Lee, H. Kim, A. Sarkar, M. K. Nazeeruddin, M. Grätzel, and S. I. Seok, Nat. Photonics 7, 486 (2013).

${ }^{20}$ P. Wang, F. Cai, L. Yang, Y. Yan, J. Cai, H. Wang, R. S. Gurney, D. Liu, and T. Wang, ACS Appl. Mater. Interfaces 10, 33144 (2018).

${ }^{21}$ A. J. Knight, J. B. Patel, H. J. Snaith, M. B. Johnston, and L. M. Herz, Adv. Energy Mater. 10, 1903488 (2020).

${ }^{22}$ C. Bracher, B. G. Freestone, D. K. Mohamad, J. A. Smith, and D. G. Lidzey, Energy Sci. Eng. 6, 35 (2018).

${ }^{23}$ K. Domanski, J.-P. Correa-Baena, N. Mine, M. K. Nazeeruddin, A. Abate, M. Saliba, W. Tress, A. Hagfeldt, and M. Grätzel, ACS Nano 10, 6306 (2016).

${ }^{24}$ See https://www.nrel.gov/grid/solar-resource/spectra-am1.5.html for information about the reference air mass 1.5 spectra.

${ }^{25}$ S. Hegedus and W. Shafarman, Prog. Photovolt. 12, 155 (2004).

${ }^{\mathbf{2 6}}$ X. Tang, M. Brandl, B. May, I. Levchuk, Y. Hou, M. Richter, H. Chen, S. Chen, S. Kahmann, A. Osvet, F. Maier, H.-P. Steinrück, R. Hock, G. J. Matt, and C. J. Brabec, J. Mater. Chem. A 4, 15896 (2016).
${ }^{27}$ S. Ito, S. Tanaka, K. Manabe, and H. Nishino, J. Phys. Chem. C 118, 16995 (2014).

${ }^{28}$ J. Chantana, T. Kato, H. Sugimoto, and T. Minemoto, Appl. Phys. Lett. 112, 151601 (2018).

${ }^{29}$ J. Chantana, Y. Kawano, T. Nishimura, and T. Minemoto, Mater. Today Commun. 21, 100652 (2019).

${ }^{30}$ J. Li, H. Wang, X. Y. Chin, H. A. Dewi, K. Vergeer, T. W. Goh, J. W. M. Lim, J. H. Lew, K. P. Loh, C. Soci, T. C. Sum, H. J. Bolink, N. Mathews, S. Mhaisalkar, and A. Bruno, Joule 4, 1 (2020).

${ }^{31}$ I. Levine, S. Gupta, A. Bera, D. Ceratti, G. Hodes, D. Cahen, D. Guo, T. J. Savenije, J. Ávila, H. J. Bolink, O. Millo, D. Azulay, and I. Balberg, J. Appl. Phys. 124, 103103 (2018).

${ }^{32}$ G. San, M. Balberg, J. Jedrzejewski, and I. Balberg, J. Appl. Phys. 127, 085103 (2020).

${ }^{33}$ A. Faroog, I. M. Hossain, S. Moghadamzadeh, J. A. Schwenzer, T. Abzieher, B. S. Richards, E. Klampaftis, and U. W. Paetzold, ACS Appl. Mater. Interfaces 10, 21985 (2018).

${ }^{34}$ D. W. deQuilettes, W. Zhang, V. M. Burlakov, D. J. Graham, T. Leijtens, A. Osherov, V. Bulović, H. J. Snaith, D. S. Ginger, and S. D. Stranks, Nat. Commun. 7, 11683 (2016).

${ }^{35}$ N. J. Jeon, J. H. Noh, Y. C. Kim, W. S. Yang, S. Ryu, and S. I. Seok, Nat. Mater. 13, 897 (2014).

${ }^{36}$ W. Chen, Y. Wu, Y. Yue, J. Liu, W. Zhang, X. Yang, H. Chen, E. Bi, I. Ashraful, M. Grätzel, and L. Han, Science 350, 944 (2015). 